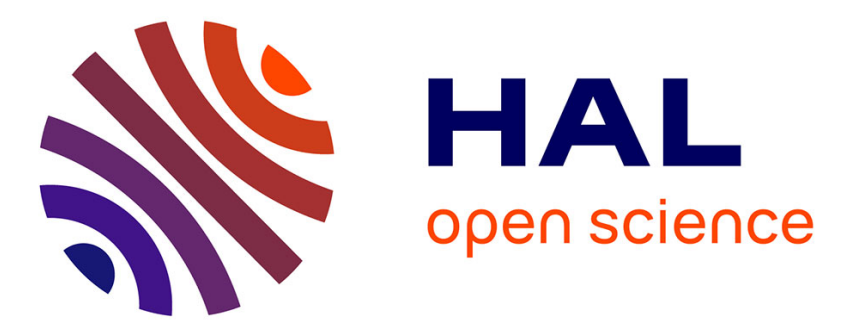

\title{
Absence of a neutralizing antibody response to humanized cobra venom factor in mice
}

Mathieu Ing, Brian E Hew, David C Fritzinger, Sandrine Delignat, Sébastien Lacroix-Desmazes, Carl-Wilhelm Vogel, Julie Rayes

\section{- To cite this version:}

Mathieu Ing, Brian E Hew, David C Fritzinger, Sandrine Delignat, Sébastien Lacroix-Desmazes, et al. Absence of a neutralizing antibody response to humanized cobra venom factor in mice. Molecular Immunology, 2018, 97, pp.1-7. 10.1016/j.molimm.2018.02.018 . hal-01832000

\section{HAL Id: hal-01832000 \\ https://hal.sorbonne-universite.fr/hal-01832000}

Submitted on 6 Jul 2018

HAL is a multi-disciplinary open access archive for the deposit and dissemination of scientific research documents, whether they are published or not. The documents may come from teaching and research institutions in France or abroad, or from public or private research centers.
L'archive ouverte pluridisciplinaire HAL, est destinée au dépôt et à la diffusion de documents scientifiques de niveau recherche, publiés ou non, émanant des établissements d'enseignement et de recherche français ou étrangers, des laboratoires publics ou privés. 


\title{
Absence of a Neutralizing Antibody Response to Humanized Cobra Venom Factor in Mice.
}

Mathieu Ing ${ }^{1}$, Brian E. Hew ${ }^{2}$, David C. Fritzinger ${ }^{2}$, Sandrine Delignat ${ }^{1}$, Sébastien Lacroix-Desmazes ${ }^{1}$, Carl-Wilhelm Vogel ${ }^{2,3}$, Julie Rayes ${ }^{1}$

${ }^{1}$ INSERM, UMRS 1138, Centre de Recherche des Cordeliers, Paris, 75006 France; ${ }^{2}$ University of Hawaii Cancer Center; ${ }^{3}$ Department of Pathology, John A. Burns School of Medicine, Honolulu, HI 96813, USA

Corresponding author: Carl-Wilhelm Vogel, University of Hawaii Cancer Center, Honolulu, HI 96813, USA. Email: cvogel@cc.hawaii.edu

Footnote:

Abbreviations: CVF, cobra venom factor; $\mathrm{nCVF}$, natural CVF as it occurs in cobra venom; rCVF, recombinantly produced CVF; hCVF, humanized CVF; huC3, human $\mathrm{C} 3$; muC3, murine $\mathrm{C} 3$.

\begin{abstract}
Cobra venom factor $(\mathrm{CVF})$ is the complement-activating protein in cobra venom. Humanized CVF (hCVF) is a human $\mathrm{C} 3$ derivative where the $\mathrm{C}$-terminal 168 amino acid residues were replaced with the homologous sequence from CVF. hCVF has been shown in multiple models of disease with complement pathology to be a promising therapeutic agent, with no observed adverse effects. Here we describe the antibody response to hCVF in two different strains of mice. hCVF was able to repeatedly decomplement the mice after four injections in weekly intervals, demonstrating the absence of a neutralizing antibody response. In contrast, natural CVF caused decomplementation in all mice only after the first administration. After two additional administrations of natural CVF, decomplementation was inconsistent and varied tremendously from mouse to mouse. After the fourth administration, natural CVF was essentially unable to deplete complement, consistent with the known generation of a neutralizing antibody response. We also analyzed the IgG antibody response to hCVF. There was great variation, with approximately one quarter of the mice exhibiting non-detectable levels of antihCVF IgG, and another quarter very low levels. The levels of anti-hCVF IgG did not correlate with the levels of remaining $\mathrm{C} 3$. The anti-hCVF antibodies cross-reacted with natural CVF, recombinant $\mathrm{CVF}$, and human $\mathrm{C} 3$. Whereas overall the level of anti-hCVF IgG cross-reacting with human $\mathrm{C} 3$ was lower compared to $\mathrm{rCVF}$ or $\mathrm{nCVF}$, mice with higher levels of anti-hCVF IgG exhibited higher binding to $\mathrm{CVF}$ and human $\mathrm{C} 3$, excluding the possibility that higher antibody levels reflect preferential immunogenicity of CVF-specific or human C3-specific epitopes.
\end{abstract}

\section{Keywords}

Cobra venom factor; humanized cobra venom factor; complement; complement depletion; antibody response

\section{Introduction}

Cobra venom factor (CVF) is the complement-activating protein in cobra venom. It is a structural and functional analog of complement component $\mathrm{C} 3$. When CVF is added to serum, it binds Factor $\mathrm{B}$ and leads to the formation of an alternative pathway $\mathrm{C} 3 / \mathrm{C} 5$ convertase. The convertase $(\mathrm{CVF}, \mathrm{Bb})$ is physico-chemically stable, and resistant to inactivation by complement regulatory proteins Factors $\mathrm{H}$ and $\mathrm{I}$, causing rapid cleavage of $\mathrm{C} 3$ and $\mathrm{C} 5$, and resulting in the depletion of the serum complement (reviews: Vogel and Fritzinger, 2010; Vogel and Fritzinger, 2017). The functional similarity of CVF to $\mathrm{C} 3$ is corroborated by its structure. Both proteins are synthesized as single-chain pro-proteins which are subsequently processed into the mature proteins with corresponding chain structures (Fig. 1). The sequence identity between $\mathrm{CVF}$ and mammalian $\mathrm{C} 3$ is about $50 \%$, with a similarity of about $70 \%$ (Fritzinger et al., 1994). Both proteins exhibit a highly similar crystal structure with identical domains (Janssen et al., 2006, 2009; Krishnan et al., 2009). 
Ever since it had been demonstrated that CVF can be safely administered to laboratory animals (Cochrane et al., 1970), the ability of CVF to exhaustively activate, and thereby deplete, complement has been exploited in innumerable studies as a tool to study the role of complement in host defense as well as pathogenesis of disease. As a matter of fact, our knowledge of the role of complement in the pathogenesis of many diseases was established by comparing normal animals with complementdepleted animals. As complement depletion by CVF eliminates the pathogenic contribution of complement to the disease process, complement depletion has more recently been recognized as a potentially valuable therapeutic approach in diseases with complement pathogenesis (Vogel and Fritzinger, 2007, Fritzinger et al., 2008; Vogel et al., 2014). However, CVF is immunogenic, generating an antibody response that limits its usefulness for complement depletion to a single injection (Cochrane et al., 1970, Pryjma and Humphrey, 1975). After identifying that the very Cterminal part of CVF harbors the important structures for forming a stable convertase (Grunwald et al., 1993; Hew et al., 2012), we attempted to overcome the limitation of immunogenicity by creating chimeric proteins in which $\mathrm{C}$-terminal amino acid residues of human $\mathrm{C} 3$ were replaced by the homologous sequence from CVF. These chimeric proteins are human $\mathrm{C} 3$ derivatives, termed "humanized CVF" (hCVF), which exhibit rapid complement depletion in vitro and in vivo just like natural CVF (Fritzinger et al., 2009; Vogel and Fritzinger, 2010; Vogel et al., 2014). Our lead hCVF protein (termed HC3-1496) consists of human C3 in which the $168 \mathrm{C}$-terminal amino acid residues are homologous to CVF. HC3-1496 exhibits an overall protein sequence identity with human C3 of 94.3\%; and even within the $168 \mathrm{C}$-terminal residues from CVF, $44.05 \%$ are identical to human C3 (Fig. 1).

hCVF has been shown in multiple preclinical disease models with complement pathogenesis to be a promising therapeutic agent. These include age-related macular degeneration (AMD) (Fritzinger et al, 2010), myocardial and gastrointestinal ischemia reperfusion injury (Gorsuch et al., 2009; Vogel et al., 2014), ventilator-induced lung injury (Takahashi et al., 2011), collagen-induced arthritis (Fritzinger et al., 2008), paroxysmal nocturnal hemoglobinuria (PNH) (Vogel and Fritzinger, 2010), myasthenia gravis (Vogel and Fritzinger, 2014), and monoclonal antibody therapy of lymphoma (Wang et al., 2009). In three disease models (AMD, arthritis, and myasthenia gravis), multiple injections of hCVF resulted in depletion for up to 30 days, suggesting the absence of a neutralizing antibody response to hCVF. This observation was recently extended in a mouse model of hemophilia A, aimed at reducing the anti-Factor VIII immune response to treatment with recombinant Factor VIII in Factor VIII knockout mice. A significant reduction in the levels of induced anti-Factor VIII antibodies was achieved by four cycles of complement depletion with hCVF in weekly intervals (Rayes et al., 2018). Here we report that repeated injections of hCVF into Factor VIII knock-out mice, as well as wild-type mice, lead to repeated complement depletion; and although antibodies are generated against hCVF, crossreacting with natural CVF, recombinant $\mathrm{CVF}$, and human $\mathrm{C}$, they do not neutralize the complementdepleting activity of hCVF.

\section{Material and methods}

Materials. Restriction enzymes and calf intestinal phosphatase were from New England Biolabs (Beverly, MA). T4 DNA ligase was either from New England Biolabs, or from Invitrogen (Carlsbad, CA). Oligonucleotides were obtained from Integrated DNA Technologies (Coralville, IA). Fastart TAQ DNA polymerase and Fastart High Fidelity DNA polymerase were obtained from Roche Applied Sciences, Inc (Indianapolis, IN). The D. melanogaster S2 expression plasmid, pMT/Bip-V5HisA, all Drosophila S2 media, and fetal calf serum were from Invitrogen. Human complement protein C3 was purchased from Merck Millipore (Merck Chemicals Ltd, Nottingham, United Kingdom). Natural CVF was purified from lyophilized Naja kaouthia venom (Miami Venom Laboratories, Punta Gorda, Florida, USA) as described (Vogel and Müller-Eberhard, 1984). Recombinant CVF (rCVF) was produced in D. melanogaster $\mathrm{S} 2$ cells as described (Kock et al., 2004). rCVF is a mixture of C3-like and C3b-like proteins (Kock et al., 2004) (Fig.1). Polyclonal goat antimouse C3 antibodies were purchased from MP Biomedicals (Illkirch, France). Biotinylated polyclonal goat anti-mouse $\mathrm{C} 3$ antibodies and streptavidin-conjugated horseradish peroxidase (HRP) were from 
R\&D systems (Lille, France). HRP-coupled polyclonal goat anti-mouse IgG antibody was from Southern Biotech (Anaheim, CA).

Preparation of the plasmid expressing hCVF protein HC3-1496. This plasmid was prepared in a manner very similar to the method described earlier for the preparation of the plasmid expressing hCVF protein HC3-1348 (pMB-HC3-1348) (Fritzinger et al., 2009). Initially, two PCR reactions were performed to obtain the human $\mathrm{C} 3$ and $\mathrm{CVF}$ portions of the coding sequence, the first using pBS$\mathrm{HuC3}$ (2) as a template and HuC3H5-3-F1 (TCTGTGTGGCAGACCCCTTCGAGG) and HuC3H5-4R1(2) (GAGAAGGCCTGTTCCTTTATCCGGATGGTAGAACCGGGTAC) as primers, and the second using pCVF-FL3 $\Delta$ as a template and HuC3H5-4-F2(2) (CCGGTTCTACCATCCGGATAAAGGAACAGGCCTTC) and HuC3H5-3-R2

(CATCCATGACATAGATATCATTACCATCTTG) as primers. Following the PCR reaction, the products were purified using the Qiagen PCR purification kit, and combined in an overlap extension PCR reaction, using the two PCR fragments as templates and HuC3H5-3-F1 and HuC3H5-3-R2 as primers. This PCR product was purified, cut with BstBI and gel purified as described above. It was then ligated into pHC3-1550(-sig) (Fritzinger et al. 2009) that had been BstBI cut and dephosphorylated with calf intestinal phosphatase. Orientation of the inserts was determined by EcoRI digestion, and clones with the inserts in the correct orientation were sequenced to ascertain the correct sequence with a lack of PCR-induced mutations. The resulting plasmid was called pHC3-1496. The HC3-1496 coding sequence was amplified by PCR. There were two amplifications, one to produce a fragment coding for HC3-1496 with the native human C3 signal sequence, and one coding for HC31496 with a mouse $\mathrm{IgG}$ signal sequence. For producing the coding sequence with the native C3 signal, the following primers were used:

PNAtf: 5'-gcaagcttGCCGCCACCATGGGACCCACCTCAGGTC-3' and Pnatr: 5'ccgcggcegcTTAAGTAGGGCAGCCAAACTCAGTCAAT-3'. The primers used for producing the HC3-1496 with the mouse IgG signal sequence were Pmsp: 5'gcaagcttGCCGCCACCATGGAGACCGACACACTGCTGCTGTGGGTGCT GCTGCTGTGGGTCCCCGGCTCCACTGGAAGTCCCATGTACTCTATCATCACC CCCAAC-3' and Pnatr: 5'-ccgcggccgcTTAAGTAGGGCAGCCAAACTCAGTCAAT-3'. The resulting PCR products were gel purified and isolated from the gel using the Qiagen QIAquick Gel Extraction Kit, and cut with HindIII and NotI. The fragments were then cloned into pOptiVEC-TOPO that had been cut with the same enzymes. The expression vectors were called pOptiVEC-1496(sig)-3\# and pOptiVEC-1496-3\# respectively.

Expression and purification of HC3-1496. pOptiVEC-1496(sig)-3\# and pOptiVEC-1496-3\# were transfected into $\mathrm{CHO}$ cells by electroporation. Following transfection, stably transfected cells were selected using either $200 \mathrm{nM}$ or $500 \mathrm{nM}$ methotrexate in liquid media. High-producing clones were then selected by plating between 2,500 and 10,000 cells onto semisolid media containing a fluorescent anti-CVF antibody in petri dishes. Colonies were allowed to grow for 10 days, and colonies producing HC3-1496 were detected, either by detecting fluorescent colonies, or colonies with a ring of immunoprecipitates. Colonies producing high yields of HC3-1496 were selected, and plaque purified. The HC3-1496 protein was purified by a combination of Capto-Q, Butyl FF HiTrap, and Q-HP HiTrap chromatography. Purified HC3-1496 is a mixture of C3-like and C3b-like proteins (Fritzinger et al., 2009).

Animals and in vivo complement depletion. Wild-type C57B1/6 mice (Janvier, Saint-Berthevin, France) and Factor VIII exon 16-invalidated C57Bl/6 mice (gift from Prof. H.H. Kazazian, University of Pennsylvania School of Medicine, Philadelphia, PA, USA) (Bi et al., 1995) were handled at 8-12 weeks-old, in agreement with French ethical authorities (authorization \#23BA53). Complement depletion was performed by intraperitoneal injections of $\mathrm{nCVF}$ or hCVF in mice $(1 \mathrm{mg} / \mathrm{kg}$, diluted in PBS), once a week for 4 consecutive weeks. Retro-orbital sampling was performed to collect blood once a week for four weeks three hours after each hCVF injection, and EDTA $(50 \mathrm{mM})$ was added to the blood samples to prevent ex vivo complement activation. Plasma was isolated from the blood and kept at $-80^{\circ} \mathrm{C}$ until use. 
Measurement of circulating $\mathbf{C 3}$ in mouse plasma. Ninety-six-well flat-bottomed ELISA plates (Nunc Maxisorp ${ }^{\circledR}$, Roskilde, Denmark) were coated with a polyclonal goat anti-mouse C3 antibody at $3.3 \mu \mathrm{g} / \mathrm{ml}$ in bicarbonate buffer, $\mathrm{pH} 9.5$, overnight at $4^{\circ} \mathrm{C}$. Wells were then blocked in PBS, $0.1 \%$ Tween-20 and 3\% bovine serum albumin (BSA), $\mathrm{pH}$ 7.4. Plasma were serially diluted in blocking buffer and added to anti-C3 coated wells for 1 hour at $37^{\circ} \mathrm{C}$. Bound $\mathrm{C} 3$ was revealed using a biotinylated polyclonal goat anti-mouse $\mathrm{C} 3$ antibody $(1.6 \mu \mathrm{g} / \mathrm{ml})$, followed by streptavidin conjugated to horseradish peroxidase using o-phenylenediamine dihydrochloride (OPD) as substrate. Absorbance was measured at $492 \mathrm{~nm}$. Serially diluted normal plasma was used as standard and considered at $100 \%$ $\mathrm{C} 3$ level. The level of residual $\mathrm{C} 3$ in mice was expressed as a percentage of the value in normal plasma. Although cross-reactivity between CVF and antisera to mammalian C3 is very weak (Vogel et al., 1984), we ensured that the measurements of plasma C3 levels after injection with nCVF or hCVF were not altered by any potential cross-reaction (see Supplementary Fig.1).

Titration of anti-human C3, anti-rCVF, anti-hCVF, and anti-nCVF IgG. ELISA plates were coated with human $\mathrm{C} 3$ (hC3), hCVF, nCVF, and $\mathrm{rCVF}(10 \mathrm{nM})$ in bicarbonate buffer, $\mathrm{pH} 9.5$, overnight at $4^{\circ} \mathrm{C}$. After blocking with PBS, $0.1 \%$ Tween-20 and 3\% BSA, pH 7.4, serially diluted plasma was incubated for 1 hour at $37^{\circ} \mathrm{C}$. Bound IgG were revealed using a HRP-coupled polyclonal goat anti-mouse $\operatorname{IgG}$ antibody and the OPD substrate. Absorbance was then measured at $492 \mathrm{~nm}$.

Statistical analysis. Statistical significance was assessed using the double-sided nonparametric MannWhitney test.

\section{Results}

Repeated administration of hCVF causes repeated depletion of complement in vivo. In order to assess the efficiency of hCVF for repeated complement depletion in mice, we compared complement C3 depletion following weekly injections of humanized (hCVF) or natural CVF (nCVF) in mice. This experiment was performed in Factor VIII knock-out mice, a preclinical model of hemophilia A, a pathology in which we have observed an important role of complement in the elicitation of anti-Factor VIII antibodies (Rayes et al., 2018). The first injection of either hCVF or nCVF resulted in rapid and drastic complement depletion in all animals as measured by the residual mouse $\mathrm{C} 3$ (muC3) levels in plasma. Indeed, circulating muC3 levels decreased from 100\% to 6.4\% [4.5-11.4\%] (median with [interquartile range]) for nCVF (Fig. 2A) and to 19.5\% [8.3-27.8\%] for hCVF (Fig. 2B), with significant mouse-to-mouse variation. Figure 3 shows a time course of $\mathrm{C} 3$ depletion after a single injection of hCVF in Factor VIII knock-out mice, demonstrating that the absence of Factor VIII does neither affect the rapid depletion of $\mathrm{C} 3$ nor its return to normal levels after one to two days as shown previously for wild-type mice and other species (Vogel and Fritzinger, 2010). Subsequent weekly injections of hCVF consistently caused efficient complement depletion to residual levels of C3 of about 20\% [14-27\%], no different from what was seen after the first injection, even at week four (Fig. 2B). In contrast, second and third weekly administrations of nCVF failed to deplete complement in a consistent manner, with resulting $\mathrm{C} 3$ levels varying greatly between normal and essentially fully depleted (Fig. 2A); and only a fourth weekly administration rendered nCVF essentially inactive (Fig. $2 \mathrm{~A}$ ). These results strongly suggest that repeated administration of hCVF, in contrast to nCVF, does not elicit a neutralizing antibody response.

\section{Repeated administration of hCVF induces a non-neutralizing humoral immune response in} mice. To exclude the remote possibility that the absence of Factor VIII affected the antibody response to hCVF, we repeated the four weekly injection schedule with hCVF in C57Bl/6 wild-type mice. As previously observed in Factor VIII-deficient mice, injections of hCVF in wild-type mice resulted in a significant reduction of circulating C3 level: 43.9\% [30.0-53.4] (Fig. 4A), again with significant mouse-to-mouse variation. After four weeks, we also determined the presence of anti-hCVF IgG antibodies. Mice receiving hCVF treatment developed anti-hCVF antibodies, as compared to the PBS control group (0.236 [0.115-0.507] AU vs 0.052 [0.049-0.056] AU, respectively) (Fig. 4B). However, there was significant variation from mouse to mouse, with approximately one quarter of the animals exhibiting essentially non-detectable antibody levels, and another quarter very low levels (Fig. 4B). 
Moreover, the anti-hCVF IgG levels in individual mice did not correlate with the levels of remaining muC3 $\left(\mathrm{r}^{2}=0.0440, \mathrm{p}=0.3750\right)$ (Fig. $\left.4 \mathrm{C}\right)$. These results suggest that hCVF exhibits an overall low degree of immunogenicity in mice, and that anti-hCVF IgG produced in mice lack neutralizing activity toward hCVF.

Anti-hCVF antibodies recognize antigenic epitopes on both CVF and human C3. hCVF protein HC3-1496 is a human C3 derivative with nCVF-like functions, obtained by replacing a short region of the C-terminus of the human C $3 \alpha$-chain with the homologous sequence from nCVF (Fig.1). The protein sequence of the chimeric single-chain pro-protein HC3-1496 is $94.3 \%$ identical to human C3; this includes the 168 residues long CVF portion of the protein where $44.05 \%$ of the residues are identical to human C3. hCVF protein HC3-1496, like other hCVF proteins (Fritzinger et al., 2009) and recombinant CVF (rCVF) (Kock et al., 2004), is usually expressed as a mixture of a C3-like and C3b-like form (Fig.1). In order to identify the immunogenic regions of hCVF in mice, we assessed the cross-reactivity of the anti-hCVF antibodies with nCVF, rCVF, and human $\mathrm{C} 3$ (huC3). The anti-hCVF antibodies recognized $\mathrm{rCVF}$ and $\mathrm{nCVF}$ similarly to hCVF (Fig. 5A, B). Anti-hCVF antibodies also bound to huC3, although many mice had essentially non-detectable levels of anti-hCVF antibodies cross-reacting with huC3 (Fig. 5C). The mean level of anti-hCVF antibodies cross-reacting with huC3 was significantly lower compared to hCVF, albeit only at a $p$-value of 0.02 .

There was a highly significant correlation when the levels of anti-hCVF were plotted against the levels of anti-nCVF, anti-rCVF, and anti-huC3 antibodies ( $p<0.0001$ in each case; Fig. 5D, E, F), indicating that mice with higher overall levels of anti-hCVF antibodies also showed increased binding to both $\mathrm{CVF}$ and huC3 epitopes, excluding the possibility that the higher antibody levels reflect preferential immunogenicity of CVF-specific or huC3-specific epitopes.

As shown above for anti-hCVF (Fig 4C), the levels of anti-nCVF, anti-rCVF, and anti-huC3 did not display any significant correlation with the levels of remaining circulating $\mathrm{C} 3$ in individual mice after the fourth injection with hCVF (Fig. 5G, H, I). This observation is consistent with the fact that the antibodies elicited against hCVF do not interfere with its C3-depleting activity, and are devoid of a neutralizing function toward hCVF.

\section{Discussion}

Humanized CVF (hCVF) is a term coined for human $\mathrm{C} 3$ derivatives where the $\mathrm{C}$-terminal portion of the $\mathrm{C} 3 \alpha$-chain has been exchanged with the homologous sequence from CVF, thereby introducing the CVF-specific functions of forming a physico-chemically stable $\mathrm{C} 3$ convertase, and exhibiting resistance to inactivation by the regulatory proteins Factors $\mathrm{H}$ and I. These two properties of hCVF, like natural CVF from cobra venom, are a prerequisite for continuous $\mathrm{C} 3$ activation, leading to complement depletion both in vitro and in vivo (Fritzinger et al., 2009; Vogel et a., 2014; Vogel and Fritzinger, 2017). hCVF was developed as a biopharmaceutic for therapeutic complement depletion in diseases where the complement system is involved in the disease pathogenicity. The efficacy of complement depletion with hCVF was subsequently demonstrated in multiple animal models of disease (Vogel et al., 2014; Rayes et al., 2018).

CVF has been used for well over four and a half decades for safe complement depletion in laboratory animals, from mice to primates. The sole adverse effect known is an acute but fleeting pulmonitis (Till et al., 1987; Mulligan et al., 1996), observed only after massive complement activation in plasma by $\mathrm{nCVF}$. The nCVF-dependent convertase not only cleaves $\mathrm{C} 3$ but also $\mathrm{C} 5$. The resulting massive generation of C5a overwhelms the body's protective mechanisms, leading to C5a-mediated neutrophil activation with subsequent sequestration to the lungs and causing pulmonitis. In contrast to $\mathrm{nCVF}$, the convertase formed with hCVF does not cleave C5 (Vogel and Fritzinger, 2010; Vogel et al., 2014). Accordingly, complement depletion with hCVF does not generate $\mathrm{C} 5 \mathrm{a}$; and even intra-arterial injection into the pulmonary artery of cynomolgus monkeys had no effect on pulmonary function. Consistent with the decades-long experience of safe complement depletion with $\mathrm{nCVF}$, and the 
absence of C5a generation by hCVF, no adverse effects of complement depletion with hCVF were seen in any of the animal models of disease (Vogel et al., 2014).

In addition to efficacy and safety, immunogenicity is an important property of any biotherapeutic. We found that hCVF efficiently depletes complement in mice after each of four weekly administrations. In contrast, $\mathrm{nCVF}$ resulted in consistent and efficient complement depletion only after the first administration. Subsequent injections over two weeks resulted in inconsistent complement depletion, with significant variation from mouse to mouse. A fourth injection rendered nCVF essentially inactive. These results confirm earlier studies that demonstrated that CVF is a potent immunogen, eliciting neutralizing antibodies (Cochrane et al., 1970; Pryjma and Humphrey, 1974), and that nCVF is a reliable reagent for complement depletion in nCVF naïve animals only. Our finding that hCVF exhibits effective complement depletion after repeated administration indicates that hCVF does not induce a neutralizing antibody response, at least after multiple injections over a four-week period.

There is always a significant variation from animal to animal, both with regard to the initial complement levels (Fig. 4A) and the extent of complement depletion (Figs. 2B, 3, 4A). We have no experimental evidence, or any explanation, why complement depletion with nCVF would differ from hCVF, and that the absence of Factor VIII would affect the complement depletion by nCVF or hCVF. Although means and interquartile ranges were somewhat different, $25 \%$ of the remaining $\mathrm{C} 3$ levels in wild-type mice after depletion with hCVF (Fig. 4A) were at the same level of Factor VIII knock-out mice (Fig. 2B), and that many Factor VIII knock-out mice after depletion with hCVF exhibited similarly low remaining $\mathrm{C} 3$ levels as seen after depletion with nCVF (compare Figs. 2B and 3 with Fig. 2A).

We analyzed the antibody response to hCVF after four weekly administrations. We detected antihCVF IgG antibodies in the murine plasma. However, the overall antigenicity of hCVF appears to be low. There was significant variation in the antibody levels of individual mice, with approximately one quarter of the animals exhibiting essentially non-detectable antibody levels, and another quarter very low levels. The anti-hCVF antibodies cross-reacted with nCVF and $\mathrm{rCVF}$ as well as huC3, indicating that both CVF-specific epitopes and huC3-specific epitopes of hCVF contribute to the immunogenicity of hCVF in mice. Our data do not allow to locate antigenic epitopes on the hCVF protein. Based on primary protein sequence differences to murine $\mathrm{C} 3$, there are CVF-specific residues in the human $\mathrm{C} 3$ portion of hCVF, and huC3-specific residues in the nCVF-portion of hCVF.

However, the number of mouse plasma with very low to non-detectable antibodies cross-reacting with human $\mathrm{C} 3$ was much lower compared to plasma cross-reacting with $\mathrm{rCVF}$ and $\mathrm{nCVF}$, suggesting that CVF-specific sequence differences are more immunogenic despite the fact that there are significantly more huC3-specific sequence differences. On the other hand, we found that mice with higher overall levels of anti-hCVF antibodies also showed increased binding to both CVF and huC3 epitopes, thereby excluding the possibility that the higher antibody levels reflect preferential immunogenicity of CVF-specific or huC3-specific epitopes.

Although mice mount an antibody response to hCVF, our data demonstrate that these antibodies are not neutralizing the complement-depleting activity of hCVF. This is further supported by the observation that there was no correlation between anti-hCVF antibody levels and remaining C3 levels in complement-depleted mice. There are likely several explanations for the low immunogenicity of hCVF and the lack of neutralizing antibodies. For one, the protein sequence of HC3-1496 is highly homologous to murine $\mathrm{C} 3$ (approximately $78 \%$ identity in the large human $\mathrm{C} 3$ portion, and approximately $43 \%$ identity within the C-terminal 168 residues). Moreover, hCVF, just like nCVF and the $\mathrm{C} 3$ proteins of all mammals, must contain the identical protein structures required for binding to Factor $\mathrm{B}$ and forming a convertase. These include the $\mathrm{C} 345 \mathrm{C}$ domain at the $\mathrm{C}$-terminus of $\mathrm{C} 3$ which binds the $\mathrm{Bb}$ portion of Factor $\mathrm{B}$, and other domains of $\mathrm{C} 3$, in particular the the $\mathrm{CUB}^{\mathrm{g}}$ domain, which is required for the initial contact with the Ba portion of Factor B, first described by us (O'Keefe et al., 1988), and subsequently confirmed by crystallography of the CVF,B complex (Janssen et al., 2009). These structures of $C 3$ are therefore highly conserved throughout the mammals (and likely all 
vertebrates), and consequently not immunogenic. Lastly, just like complement depletion with hCVF reduces the antibody response to Factor VIII (Rayes et al., 2018), the hCVF-induced complement depletion may aid in lowering its own immunogenicity.

Our results were obtained by injecting hCVF into mice. It may therefore not be unreasonable to speculate that hCVF might be even less immunogenic in humans as the total number of different amino acids between $\mathrm{HC} 3-1496$ and murine $\mathrm{C} 3$ will be reduced from approximately 400 to less than 100 residues between HC3-1496 and human C3, all within the 168 amino acid long C-terminal sequence only. Moreover, the three-dimensional structure of the $\mathrm{C}$-terminal sequence has been predicted by in silico analysis to be essentially identical between human C3 and hCVF (Vogel et al., 2008; Vogel et al., 2014). However, immunogenicity is not only determined by primary structure differences, and complement depletion by hCVF in itself may affect an antibody response differently in humans than in mice. Ultimately, the degree of immunogenicity of hCVF in humans is unpredictable, and will have to await clinical trials.

In conclusion, hCVF is a promising therapeutic agent for the many diseases with complement pathogenesis. It has been shown to be efficacious in multiple animal disease models, it has no known adverse effects, and as shown here, it does not induce neutralizing antibodies in mice, at least not after multiple injections over a one-month period. The effect of longer-term complement depletion with hCVF on the risk of developing infections is unknown. However, complement depletion with hCVF in never complete. There is always a significant amount of $\mathrm{C} 3$ remaining, along with normal levels of $\mathrm{C} 5$ and the other terminal complement components, ensuring some protection against infections. This is convincingly demonstrated by CVF-transgenic mice which exhibit a normal life span and, in contrast to C3 knock-out mice, no tendency to develop infections (Andrä et al., 2002; Fritzinger et al., 2010).

\section{Acknowledgments}

This study was supported by Institut National de la Santé et de la Recherche Médicale (INSERM), Centre National de la Recherche Scientifique (CNRS), Université Pierre et Marie Curie (UPMC) Paris 6, by a grant from Pfizer (Aspire Haemophilia research award 2016 WI212828), by a grant from Incode Corporation, San Diego, CA, and by funds from the University of Hawaii Cancer Center. MI was the recipient of a fellowship from Ministère de l'enseignement supérieur et de la recherche.

\section{Authors' contributions}

Designed research: MI, SLD, CWV, JR

Performed experiments: MI, BEH, DCF, SD, JR

Analyzed data: MI, SLD, CWV, JR

Wrote the paper: MI, SLD, CWV, JR

\section{References}

Andrä, J., Halter, R., Kock, M.A., Niemann, H., Vogel, C.-W., Paul, D. (2002). Generation and characterization of transgenic mice expressing cobra venom factor. Mol. Immunol. 39: 357-365.

Bi, L., Lawler, A.M., Antonarakis, S.Ee, High, K.A., Gearhart, J.D., Kazazian, Jr., H.H. (1995). Targeted disruption of the mouse factor VIII gene produces a model of haemophilia A. Nature Genet. 10: 119-121.

Cochrane, C.G., Müller-Eberhard, H.J., Aikin, B.S. (1970). Depletion of plasma complement in vivo by a protein of cobra venom: Its effect on various immunologic reactions. J. Immunol. 105: 55-69.

Eggertsen, G., Lind, P., Sjöquist, J. (1981). Molecular Characterization of the complement activating protein in the venom of the Indian cobra (Naja n. siamensis). Mol. Immunol. 18: 125-133. 
Eggertsen, G., Lundwall, Å., Hellman, U., Sjöquist, J. (1983). Antigenic relationships between human and cobra complement factors $\mathrm{C} 3$ and cobra venom factor (CVF) from the Indian cobra (Naja naja). J. Immunol. 131: 1920-1923.

Fritzinger, D.C., Bredehorst, R., Vogel, C.-W. (1994). Molecular cloning and derived primary structure of cobra venom factor. Proc. Natl. Acad. Sci. USA 91: 12775-12779.

Fritzinger, D.C., Dean, R., Meschter, C., Wong, K., Halter, R., Borlak, J., St. John, W.D., Vogel, C.W. (2010). Complement depletion with humanized cobra venom factor in a mouse model of agerelated macular degeneration. Adv. Exp. Med. Biol. 703: 151-162.

Fritzinger, D.C., Hew, B.E., Lee, J.Q., Newhouse, J, Alam, M., Gorsuch, W.B., Guikema, B.J., Stahl, G.L, Ciallella, J.R., Bowers, M., Vogel, C.-W. (2008). Derivatives of human complement component C3 for therapeutic complement depletion: A novel class of therapeutic agents. Adv. Exp. Med. Biol. 632: 293-307.

Fritzinger, D.C., Hew, B.E., Thorne, M., Pangburn, M.K., Janssen, B.J., Gros, P., Vogel, C.-W. (2009). Functional characterization of human C3/cobra venom factor hybrid proteins for therapeutic complement depletion. Dev. Comp. Immunol. 33: 105-116.

Gorsuch, W.B., Guikema B.J., Fritzinger D.C., Vogel C.-W., Stahl G.L. (2009). Humanized cobra venom factor decreases myocardial ischemia-reperfusion injury. Mol Immunol. 47: 506-510.

Grunwald, T., Ziegelmüller, P., Ollert, M.W., Bredehorst, R., Vogel, C.-W. (1993). Cobra venom factor: An intact $\beta$-Chain is not required for activity. —-Mol. Immunol. 30, Suppl. 1: 13.

Hew, B.E., Wehrhahn, D., Fritzinger, D.C., Vogel, C.-W. (2012). Hybrid proteins of cobra venom factor and cobra $\mathrm{C} 3$ : Tools to identify functionally important regions in cobra venom factor. Toxicon 60: 632-647.

Janssen, B.J., Christodoulidou, A., McCarthy, A., Lambris, J.D., Gros, P. (2006). Structure of C3b reveals conformational changes that underlie complement activity. Nature 444: 213-216.

Janssen, B.J., Gomes, L., Koning, R.I., Svergun, D.I., Koster, A.J., Fritzinger, D.C., Vogel, C.-W., Gros, P. (2009). Insights into complement convertase formation based on the structure of the factor Bcobra venom factor complex. EMBO J. 28: 2469-2478.

Kock, M.A., Hew, B.E., Bammert, H., Fritzinger, D.C., Vogel, C.-W. (2004). Structure and function of recombinant cobra venom factor. J. Biol. Chem. 279: 30836-30843.

Krishnan, V., Ponnuraj, K., Xu, Y., Macon, K., Volanakis, J.E., Narayana, S.V. (2009). The crystal structure of cobra venom factor, a cofactor for C3- and C5-convertase CVF,Bb. Structure 17: 611-619.

Mulligan, M.S., Schmid, E., Beck-Schimmer, B., Till, G.O., Friedl, H.P., Brauer, R.B., Hugli, T.E., Miyasaka, M., Warner, R.L., Johnson, K.J., Ward, P.A. (1996). Requirement and role of C5a in acute lung inflammatory injury in rats. J. Clin. Invest. 98: 503-512.

O’Keefe, M.C., Caporale, L.H., Vogel, C.-W. (1988). A novel cleavage product of human complement component $\mathrm{C} 3$ with structural and functional properties of cobra venom factor. J. Biol. Chem. 263: 12690-12697.

Pryjma, J., Humphrey, J.H. (1975). Prolonged C3 depletion by cobra venom factor in thymus-deprived mice and its implication for the role of $\mathrm{C} 3$ as an essential second signal for B-cell triggering.

Immunology 28: 569-576. 
Rayes, J., Ing, M., Delignat, S., Peyron, I., Gilardin, L., Vogel, C.-W., Fritzinger, D.C., FrémeauxBacchi, V., Kaveri, S.V., Roumenina, L., Lacroix-Desmazes, S. (2017). Complement C3 is a novel modulator of the anti-FVIII immune response. Haematologica, in press (Epub ahead of print: doi: 10.3324/haematol.2017.165720).

Takahashi, K., Saha, D., Shattino, I., Pavlov, V.I., Stahl, G.L., Finnegan, P., Vidal Melo, M.F. (2011). Complement 3 is involved with ventilator-induced lung injury. Intern. Immunopharmacol. 11: 21382143.

Till, G.O., Morganroth, M.L., Kunkel, R., Ward, P.A. (1987). Activation of C5 by cobra venom factor is required in neutrophil-mediated lung injury in the rat. Am. J. Pathol. 129: 44-53.

Vogel, C.-W., Finnegan, P.W., Fritzinger, D.C. (2014). Humanized cobra venom factor: Structure, activity, and therapeutic efficacy in preclinical disease models. Mol. Immunol. 61: 191-203.

Vogel, C.-W., Fritzinger, D. (2007). Humanized cobra venom factor: Experimental therapeutics for targeted complement activation and complement depletion. Curr. Pharmaceutical Design 13: 29162926.

Vogel, C.-W., Fritzinger, D.C. (2010). Cobra venom factor: Structure, function, and humanization for therapeutic complement depletion. Toxicon 56: 1198-1222.

Vogel, C.-W., Fritzinger, D.C. (2017). Cobra Venom Factor: The Unique Component of Cobra Venom that Activates the Complement System. In: Handbook of Toxinology. Snake Venoms. (P. Gopalakrishnakone, H. Inagaki, C.-W. Vogel, A.K. Mukherjee, T.R. Rahmy, Eds.), pp. 345-404. Springer Nature, Dordrecht, The Netherlands.

Vogel, C.-W., Fritzinger, D.C., Gorsuch, W.B., Stahl, G.L. (2015). Complement depletion with humanised cobra venom factor: Efficacy in preclinical models of vascular diseases. Thromb. Haemost. 113: 548-552.

Vogel, C.-W., Müller-Eberhard, H.J. (1984). Cobra venom factor: Improved method for purification and biochemical characterization. J. Immunol. Methods 73: 203-220.

Vogel, C.-W., Smith, C.A., Müller-Eberhard, H.J. (1984). Cobra venom factor: Structural homology with the third component of human complement. J. Immunol. 133: 3235-3241.

Wang, S.-Y.,Veeramani, S., Racila, A., Cagley, J., Fritzinger, D.C., Vogel, C.-W., St. John, W., Weiner, G.J. (2009). Depletion of the C3 component of complement enhances the ability of rituximabcoated target cells to activate human NK cells and improves the efficacy of monoclonal antibody therapy in an in vivo model. Blood 114: 5322-5330.

\section{Figure Legends}

Figure 1: Schematic representation of the chain structures of human C3, hCVF (HC3-1496), and CVF. Shown are the single-chain structures of pro-C3, pro-HC3-1496, and pro-CVF. The Ntermini are to the left. The chain homologies are indicated. Please note that the nomenclature of the three chains of nCVF ( $\alpha$-chain, $\beta$-chain, $\gamma$-chain) was coined before their homology to $\mathrm{C} 3$ chains was established by N-terminal sequencing (Eggertsen et al., 1981), immunologic cross-reaction (Eggertsen et al., 1983), and molecular cloning (Fritzinger et al., 1994). Both hCVF and rCVF are usually expressed as a mixture of C3-like and C3b-like proteins (Fritzinger et al., 2009; Kock et al., 2004). 
Please note that $\mathrm{rCVF}$ is fully active without processing into the three-chain structure of nCVF (Kock et al., 2004).

Figure 2. Repeated injection of hCVF, but not natural CVF, causes repeated complement depletion in mice. Factor VIII-deficient mice were injected intraperitoneally with (Panel A) nCVF $(\mathrm{n}=8)$ or (Panel B) hCVF (HC3-1496, $\mathrm{n}=24)$ at a dose of $1 \mathrm{mg} / \mathrm{kg}$, once a week for 4 consecutive weeks. Blood was collected before (Week 0) and weekly 3 hours post-CVF injection (Weeks 1-4). Residual circulating mouse C3 (muC3) level was measured by sandwich ELISA. Results are expressed as a percentage of circulating $\mathrm{C} 3$ in normal plasma, measured in mice before depletion and considered at $100 \%$. Horizontal bars represent the median of each time point.

Figure 3. Time course of $\mathrm{C} 3$ depletion with hCVF in Factor VIII knock-out mice. Factor VIII knock-out mice were injected intraperitoneally with $20 \mu \mathrm{g} \mathrm{hCVF}$. Blood was collected at different time points as indicated, and C3 levels were measured in plasma by sandwich ELISA.

Figure 4. Repeated administration of hCVF is associated with a non-neutralizing humoral immune response. Wild-type mice were injected intraperitoneally with PBS $(n=20)$ or HC3-1496 (hCVF, $\mathrm{n}=20$ ) at a dose of $1 \mathrm{mg} / \mathrm{kg}$, once a week for 4 consecutive weeks. Four weeks following the first injection, blood was collected and residual mouse $\mathrm{C} 3$ (muC3) and anti-hCVF antibody levels were measured in the plasma. Panel A. Residual muC3 levels in plasma was measured by ELISA. Results are expressed as a percentage compared to a normal C3 plasma level, considered at $100 \%$. Horizontal bars represent the median for each group. Panel B. Anti-hCVF IgG levels in plasma were assessed by ELISA. Results are expressed in optical densities measured at $492 \mathrm{~nm}$ (arbitrary units, AU). Horizontal bars represent the median for each group. Panel C. Correlation between the level of anti-hCVF IgG and the residual percentage of circulating muC3 in mice treated with hCVF. The linear regression curve is represented as a full line curve, and the $p$ and $r^{2}$ values are indicated.

Figure 5. Antibodies against hCVF recognize both CVF- and human C3-specific epitopes. Wildtype mice were injected intraperitoneally with PBS $(n=20)$ or HC3-1496 (hCVF, $n=20)$ once a week for four consecutive weeks, and blood was collected four weeks following the first injection. Panels A, B, C. Levels of anti-natural CVF (nCVF, Panel A), anti-recombinant CVF (rCVF, Panel B), and anti-human $\mathrm{C} 3$ (huC3, Panel $C$ ) IgG in the plasma were assessed by ELISA. Results are expressed as optical densities measured at $492 \mathrm{~nm}$ (arbitrary units, AU). Horizontal bars represent the median for each group. Panels D, E, F. Correlations between the levels of anti-nCVF (Panel D), anti-rCVF (Panel E), and anti-huC3 (Panel F) IgG compared to the level of anti-hCVF IgG are shown. The linear regression curves are represented as full line curves, and the corresponding $p$ and $r^{2}$ values are indicated. Panels G, H, I. Correlations between the levels of anti-nCVF (Panel G), anti-rCVF (Panel $H$ ), and anti-huC3 (Panel I) IgG and the residual circulating C3 levels in hCVF-treated mice are shown. The linear regression curves are represented as full line curves, and the corresponding $p$ and $r^{2}$ values are indicated.

\section{Supplementary Material}

To exclude the possibility that the measurements of $\mathrm{C} 3$ levels in mice after injection with nCVF or hCVF by the sandwich ELISA assay were falsely overestimated by any cross-reaction of nCVF or hCVF with the anti-muC3antibodies used in the assay, we assessed the possible cross-reaction. Supplemental Fig. 1 shows that neither nCVF, rCVF, nor hCVF of up to $150 \mu \mathrm{g} / \mathrm{ml}$ cross-reacts with the anti-muC3 antibodies employed in the assay. Given that mice (of approximately $20 \mathrm{~g}$ body weight) were injected with $20 \mu \mathrm{g} \mathrm{hCVF}$ or nCVF, $150 \mu \mathrm{g} / \mathrm{ml}$ is at least one order of magnitude above the potential peak plasma concentration. We had previously shown that the peak plasma concentration in mice after injection of $10 \mu \mathrm{g} \mathrm{hCVF}$ was $1.5 \mu \mathrm{g} / \mathrm{ml}$, two orders of magnitude below $150 \mu \mathrm{g} / \mathrm{ml}$.

\section{Figure Legend}


Supplemental Figure 1: Lack of cross-reactivity of nCVF, rCVF, and hCVF with anti-muC3. The sandwich ELISA assay for murine $\mathrm{C} 3$ was performed with serial 1:10 dilutions of $\mathrm{nCVF}\left({ }^{*}\right), \mathrm{rCVF}$ $(\boldsymbol{\nabla})$, and hCVF $(\Delta)$, starting at $150 \mu \mathrm{g} / \mathrm{ml}$. Mouse plasma from Factor VIII knock-out mice at a 1:10 dilution (with an estimated concentration of $\mathrm{C} 3$ of $150 \mu \mathrm{g} / \mathrm{ml}$ ) and serial 1:10 dilutions served as positive control $(\bullet)$. Results are expressed in optical densities measured (arbitrary units, AU). 


\section{Figure 2}

A $100-0000$
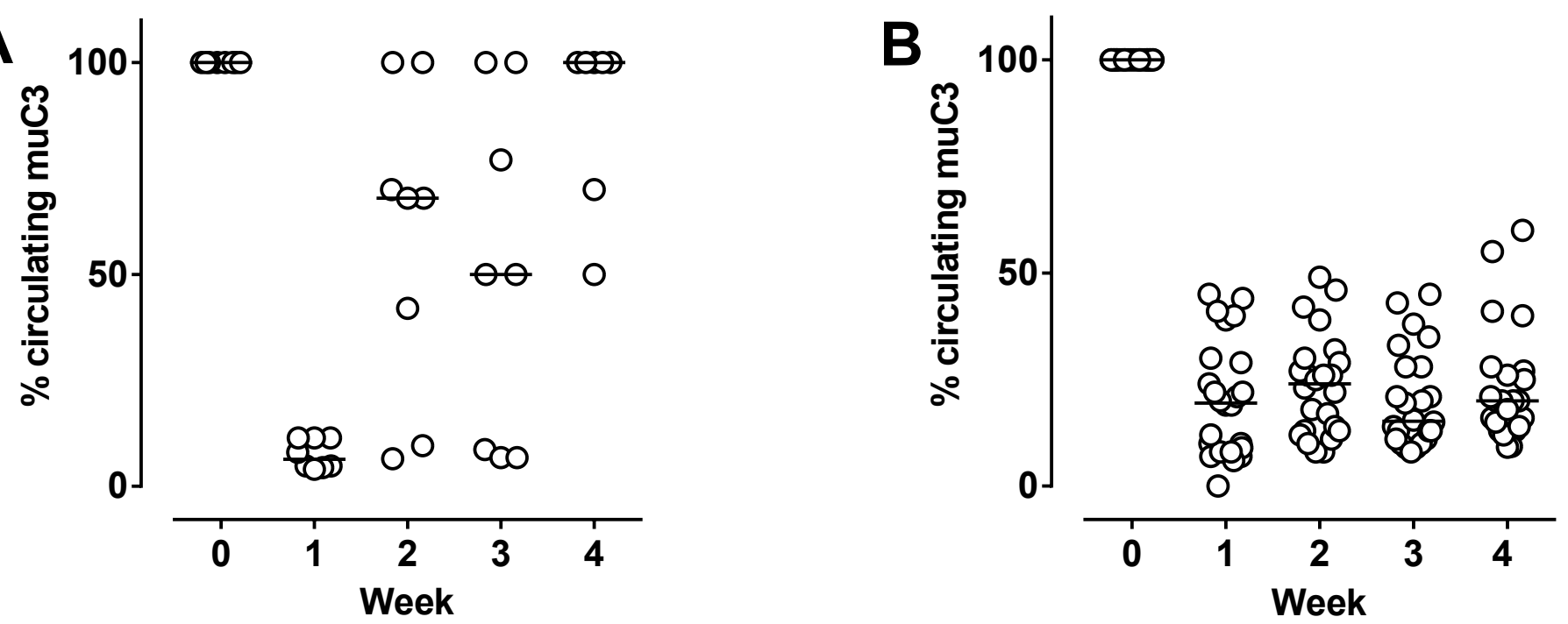
Figure

Figure 3

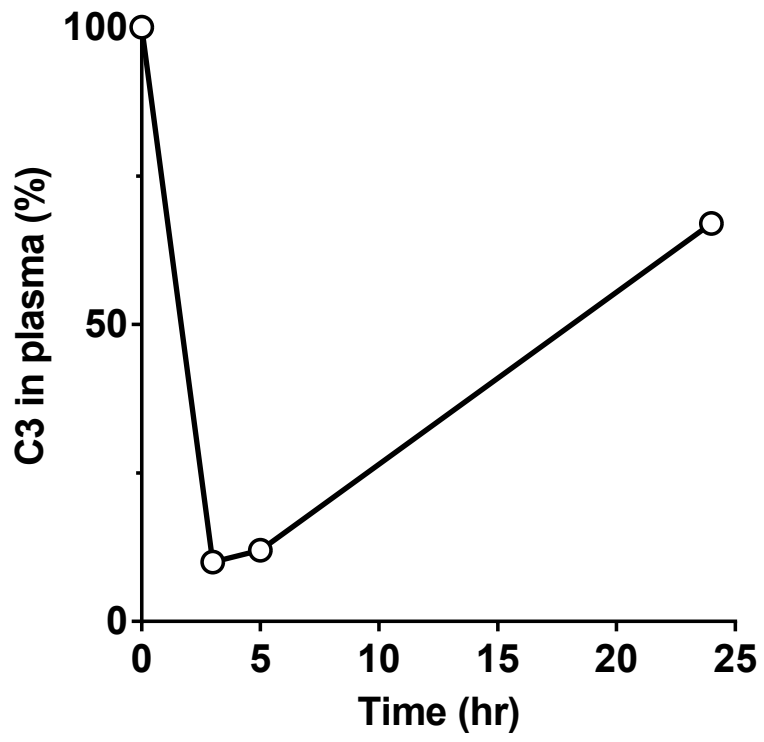


Figure 5

A

D

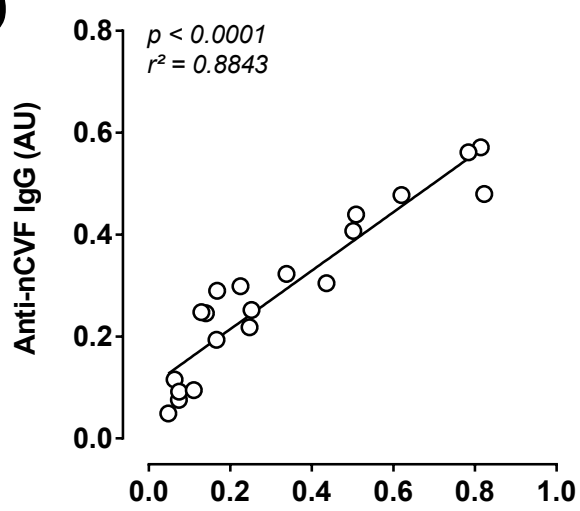

Anti-hCVF IgG (AU)

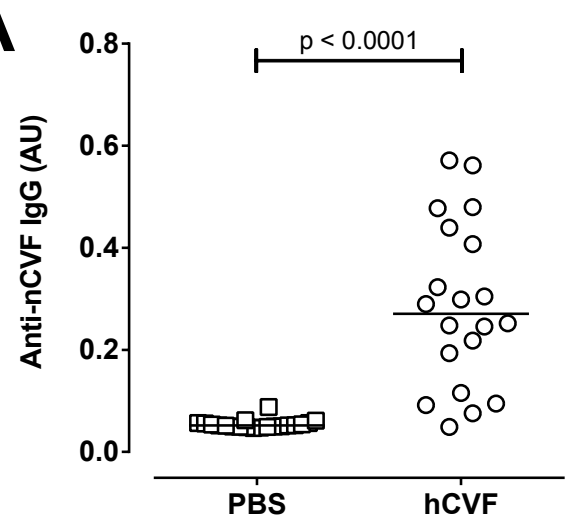

B

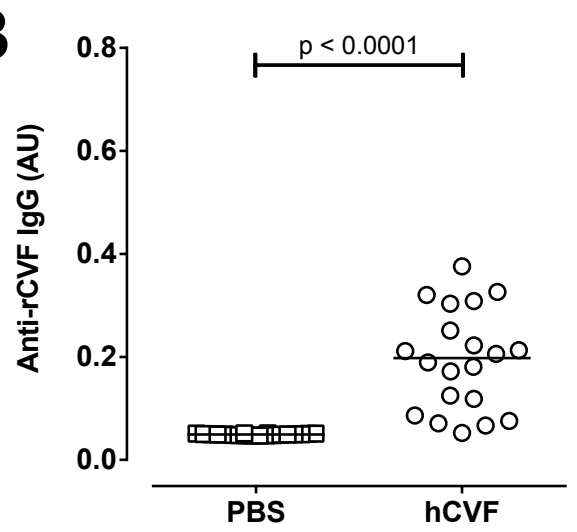

E

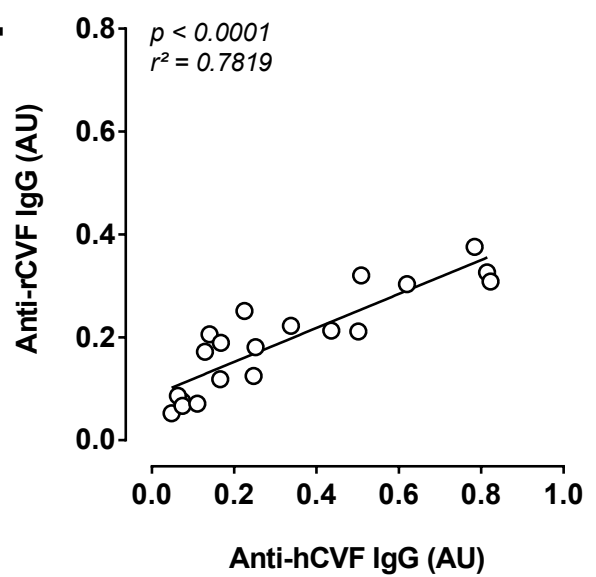

H

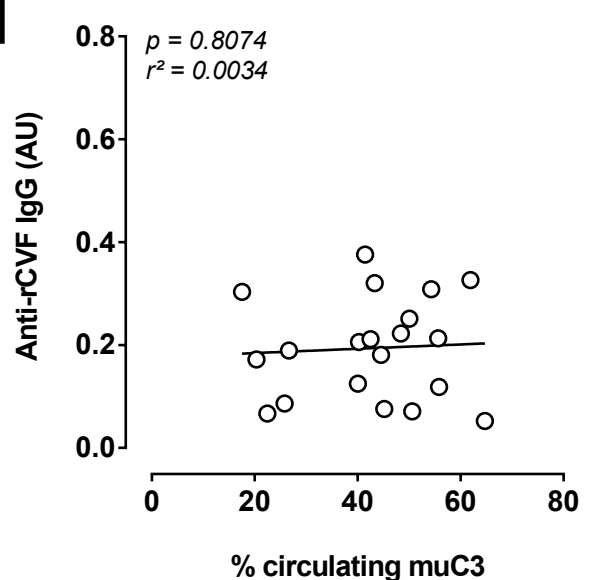

C

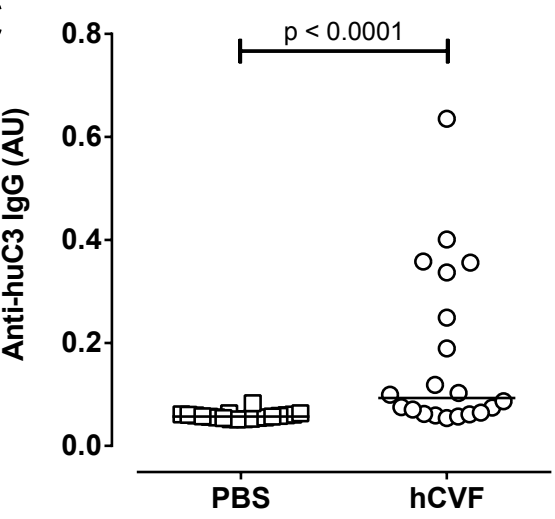

$\mathbf{F}$

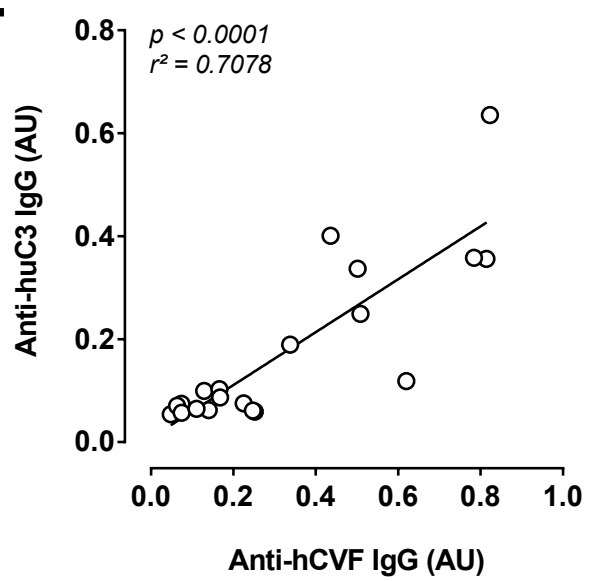

G

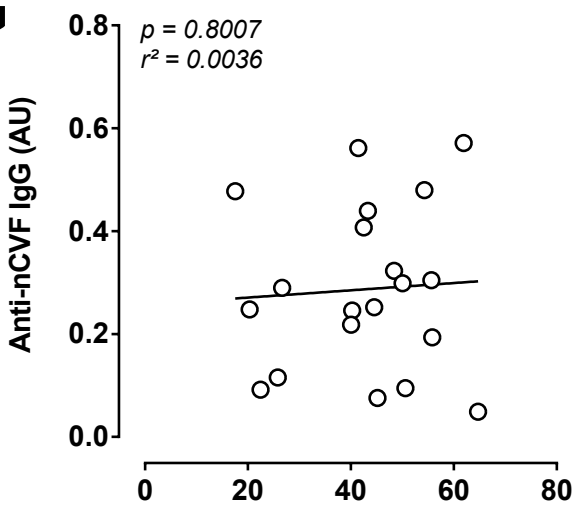

$\%$ circulating muC3

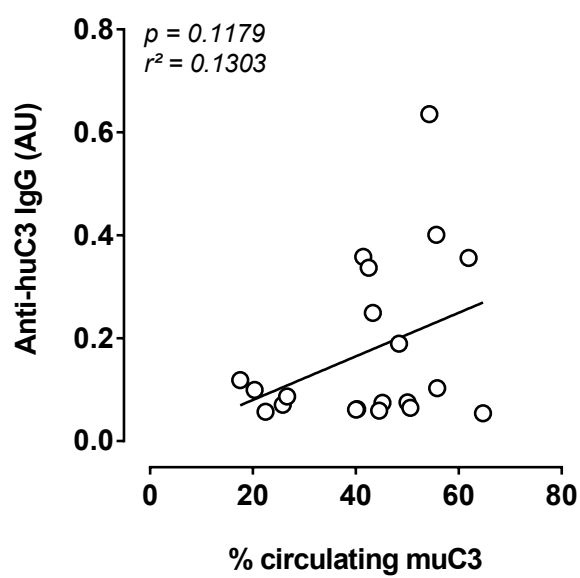




\section{Supplementary Figure 1}

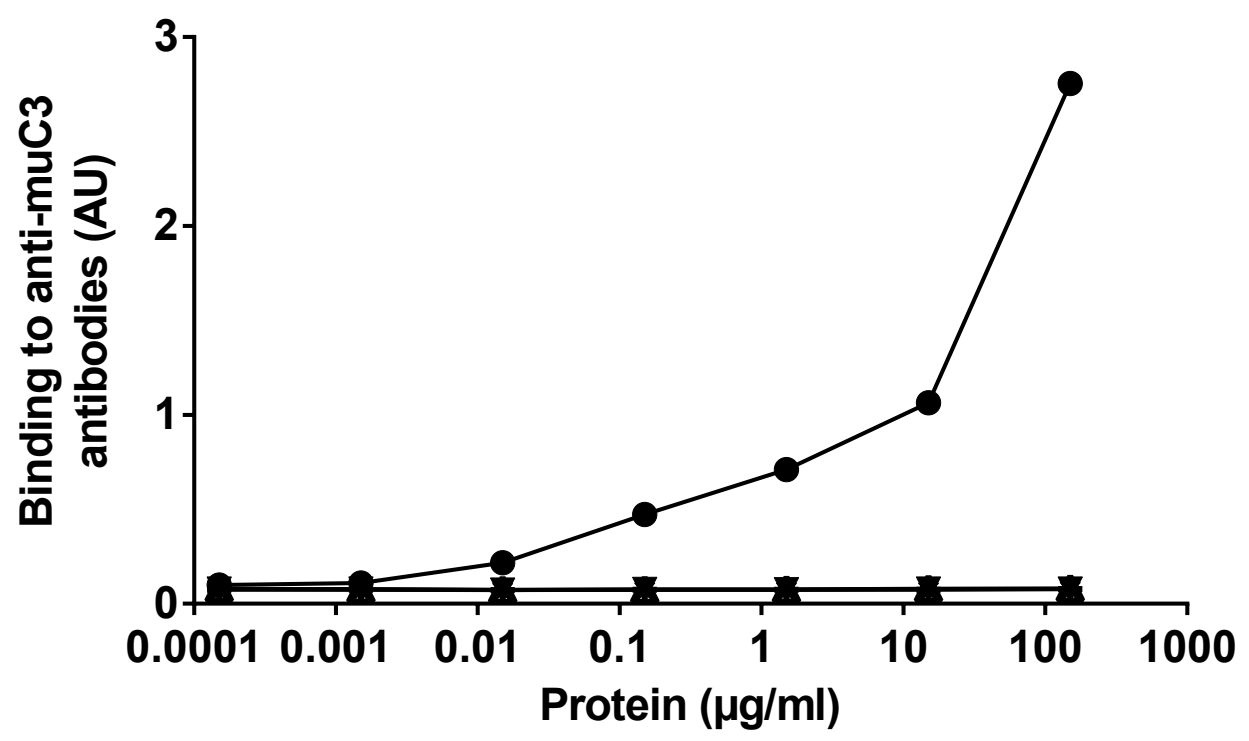

